\title{
Deciding Linear Inequalities by Computing Loop Residues
}

\author{
ROBERT SHOSTAK
}

SRI Internatınal, Menlo Park, Callfornia

\begin{abstract}
V R Pratt has shown that the real and integer feasibility of sets of linear inequalities of the form $x \leq y+c$ can be decided quickly by examining the loops in certain graphs Pratt's method is generalized, first to real feasibility of inequalities in two variables and arbitrary coefficients, and ultimately to real feasibility of arbitrary sets of linear inequalities The method is well suited to applications in program verification
\end{abstract}

KEY WORDS AND PHRASES theorem proving, deciston procedures, program verification, linear programming

CR CATEGORIES $315,369,521,532,541$

\section{Introduction}

Procedures for deciding whether a given set of linear inequalities has solutions often play an important role in deductive systems for program verification. Array bounds checks and tests on index variables are but two of the many common programming constructs that give rise to formulas involving inequalitıes. A number of approaches have been used to decide the feasibility of sets of inequalities $[3,8,9,16,22]$, ranging from goal-driven rewriting mechanisms [27] to the powerful simplex techniques [8] of linear programming. Some simple methods are well suited to the small, trivial problems that most often arise, but are insufficiently general. Full-scale simplex techniques, on the other hand, are general and fast for medium to large problems, but do not take advantage of the trivial structure of the small problems (involving only a few variables and equations) encountered most frequently in program verification and related applications.

The algorithm presented here retains the generality needed in the exceptional case, without sacrifice of speed and simplicity in the more typical small problem case. It builds on V. R. Pratt's observation $[18,20]$ that most of the inequalities that arise from verification conditions are of the form $x \leq y+c$, where $x$ and $y$ are variables and $c$ is a constant. Pratt showed that a conjunction of such inequalities can be decided quickly by examining the loops of a graph constructed from the inequalities of the conjunction. We generalize this approach, first to inequalities with no more

Permission to copy without fee all or part of this material is granted provided that the copies are not made or distributed for direct commercial advantage, the ACM copyright notice and the title of the publication and its date appear, and notice is given that copying is by permission of the Association for Computing Machinery To copy otherwise, or to republish, requires a fee and/or specific permission

This work was supported in part by the National Science Foundation under Grant MCS 76-81425, the Air Force Office of Scientific Research under Contract F44620-73-C-0068, and the Rome Air Development Center under Contract F30602-78-C-0031 A condensed version appeared in the Proceedings of the Fourth Workshop on Automatic Deduction, Austın, Texas, February 1979

Author's address SRI International, 333 Ravenswood Avenue, Menlo Park, CA 94025

(C) 1981 ACM 0004-5411/81/1000-0769\$00 75 
than two variables and with arbitrary coefficients, and then to arbitrary linear inequalities. Our generalization reduces to Pratt's test for inputs having the simple structure he describes.

The algorithm has recently been used by Aspvall and Shiloach [1] as the basis for a polynomial-time algorithm for the two-variable case.

It should be remarked that the usefulness of the algorithm for verification-type applications is not affected by the recent work of L. G. Khachiyan showing that linear programming requires polynomial time in the worst case [16]. While Khachiyan's algorithm has not yet been thoroughly evaluated (at least in the West), a number of researchers have expressed doubt as to its usefulness in practice. In any case, Khachiyan's algorithm is clearly not suited to very small problems of the kind that arise in program verification. For the examples given in this paper, the initialization step alone of Khachiyan's algorithm requires more computation than does their complete solution using the method of loop residues.

The discussion is presented in six sections. Sections 2 and 3 are concerned with preliminary definitions and a statement of the method for inequalities with two variables and arbitrary coefficients. Section 4 discusses issues of complexity and usefulness for integer problems and relates the method to Pratt's. Sections 5 and 6 deal with the extension of the method to sets having strict inequalities and sets with arbitrary linear inequalities. The last section presents a proof of the theorem that underlies the method.

\section{Definutions}

Let $S$ be a set of linear inequalities each of whose members can be written in the form $a x+b y \leq c$, where $x, y$ are real variables and $a, b, c$ are reals. Without loss of generality we require that all variables appearing in $S$ other than a special variable $v_{0}$, called the zero variable, have nonzero coefficients. We also assume that $v_{0}$ appears only with coefficient zero.

Construct an undirected multigraph $G$ from $S$ as follows. Give $G$ a vertex for each variable occurring in $S$ and an edge for each inequality. Let the edge associated with an inequality $a x+b y \leq c$ connect the vertex for $x$ with the vertex for $y$. Label each vertex with its associated variable ${ }^{1}$ and each edge with its associated inequality. $G$ is said to be the graph for $S$.

Now let $P$ be a path through $G$, given by a sequence $v_{1}, v_{2}, \ldots, v_{n+1}$ of vertices and a sequence $e_{1}, e_{2}, \ldots, e_{n}$ of edges, $n \geq 1$. The triple sequence for $P$ is given by

$$
\left\langle a_{1}, b_{1}, c_{1}\right\rangle,\left\langle a_{2}, b_{2}, c_{2}\right\rangle, \ldots,\left\langle a_{n}, b_{n}, c_{n}\right\rangle,
$$

where for each $i, 1 \leq i \leq n, a_{l} v_{\imath}+b_{\imath} v_{\imath+1} \leq c_{\imath}$ is the inequality labeling $e_{\imath}{ }^{2} P$ is admissible if, for $1 \leq \imath \leq n-1, b_{\imath}$ and $a_{\imath+1}$ have opposite signs, that is, one is strictly positive and the other is negative.

Intuitively, admissible paths correspond to sequences of inequalities that form transitıvity chains. For example, the sequence $x \leq y, y \leq z, z \leq 3$ gives rise to an admissible path, as does

$$
2 x \geq 3 y-4, \quad 2 y \geq 4-z, \quad-z \geq x .
$$

\footnotetext{
${ }^{1}$ In what follows it is notationally convenient to write $v$ for both the variable $v$ and the vertex associated with that variable

${ }^{2}$ In the case where $v_{t}$ and $v_{\imath+1}$ happen to be identical (1 e, $e_{1}$ is a self-loop), an arbitrary choice is made as to the ordering of the first two components of the associated triple
} 
Note that the sequence

$$
x \leq y, \quad y \leq z, \quad-z \leq r
$$

cannot label an admissible path, since the coefficients of $z$ have the wrong relative signs.

A path is a loop if its first and last vertices are identical. A loop is simple if its intermediate vertices are distinct.

Note that the reverse of an admissible loop is always admissible, and that the cyclic permutations of a loop $P$ are admissible if and only if $a_{1}$ and $b_{n}$ are of opposite sign, where $\left\langle a_{1}, b_{1}, c_{1}\right\rangle, \ldots,\left\langle a_{n}, b_{n}, c_{n}\right\rangle$ is the triple sequence for $P$. In this case, we say $P$ is permutable. Note also that since $\nu_{0}$ appears in $S$ only with coefficient 0 , no admissible loop with initial vertex $v_{0}$ is permutable.

Now define, for a given admissible path $P$, the residue inequality of $P$ as the inequality obtained from $P$ by applying transitivity to the inequalities labeling its edges. For example, if the inequalities along $P$ are

$$
x \leq 2 y+1, \quad y \leq 2-3 z, \quad-z \leq w,
$$

we have

$$
x \leq 2 y+1 \leq 2(2-3 z)+1 \leq 2(2+3 w)+1=6 w+5 .
$$

The residue inequality of $P$ is thus $x-6 w \leq 5$.

More formally, define the residue $r_{p}$ of $P$ as the triple $\left\langle a_{P}, b_{P}, c_{P}\right\rangle$ given by

$$
\left\langle a_{P}, b_{P}, c_{P}\right\rangle=\left\langle a_{1}, b_{1}, c_{1}\right\rangle *\left\langle a_{2}, b_{2}, c_{2}\right\rangle * \ldots *\left\langle a_{n}, b_{n}, c_{n}\right\rangle,
$$

where $\left\langle a_{1}, b_{1}, c_{1}\right), \ldots,\left\langle a_{n}, b_{n}, c_{n}\right\rangle$ is the triple sequence for $P$ and $*$ is the binary operation on triples defined by

$$
\langle a, b, c\rangle *\left\langle a^{\prime}, b^{\prime}, c^{\prime}\right\rangle=\left\langle k a a^{\prime},-k b b^{\prime}, k\left(c a^{\prime}-c^{\prime} b\right)\right\rangle \quad \text { and } \quad k=\frac{a^{\prime}}{\left|a^{\prime}\right|} .
$$

The residue inequality of $P$ is then given by $a_{P} x+b_{P} y \leq c_{P}$, where $x$ and $y$ are the first and last vertices, respectively, of $P$.

It is straightforward to show that $*$ is associative, so that $r_{P}$ is in fact uniquely defined. The idea that the residue inequality of a path is implied by the inequalities labeling the path is expressed in the following lemma.

Lemma 1. Any point (i.e., assignment of reals to variables) that satisfies the inequalities labeling an admissible path $P$ also satisfies the residue inequality of $P$.

Proof. Straightforward by induction on the length of $P$. Q.E.D.

\section{Procedure for Inequalities with Two Variables}

In the case where $P$ is a loop with initial vertex, say, $x$, Lemma 1 asserts that any point satisfying the inequalities along $P$ must also satisfy $a_{P} x+b_{P} x \leq c_{P}$. If it happens that $a_{P}+b_{P}=0$ and $c_{p}<0$, the residue inequality of $P$ is false, and we say that $P$ is an infeasible loop.

It follows that a set $S$ of inequalities is unsatisfiable if the graph $G$ for $S$ has an infeasible loop. The converse, however, does not hold in general. Figure 1, for example, shows the graph for $S=\{x \leq y, 2 x+y \leq 1, z \leq x, w \leq z, z \leq 1+w$, $\left.z \geq \frac{1}{2}\right\}$. Although $S$ is unsatisfiable, the graph has no infeasible loops, simple or otherwise.

The gist of our main theorem is that $G$ can be modified to obtain a graph $G^{\prime}$ that has an infeasible simple loop if and only if $S$ is unsatisfiable. 


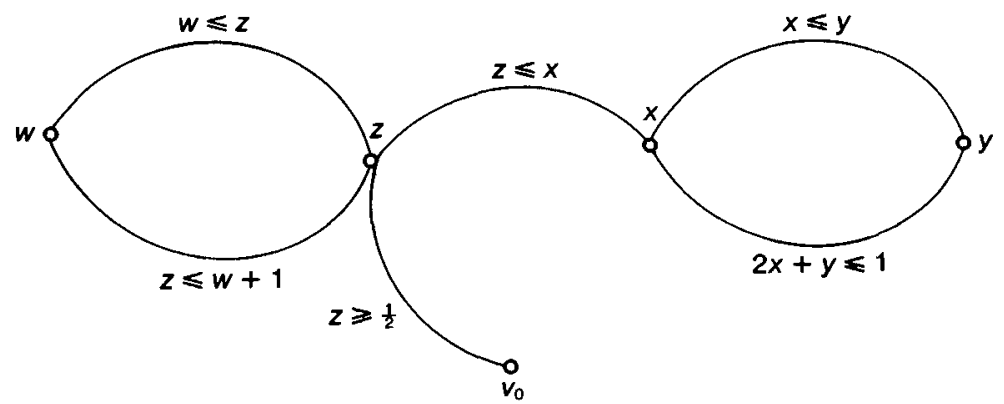

FIG. 1. Graph $G$ for $S=\left\{x \leq y, 2 x+y \leq 1, z \leq x, w \leq z, z \leq w+1, z \geq \frac{1}{2}\right\}$.

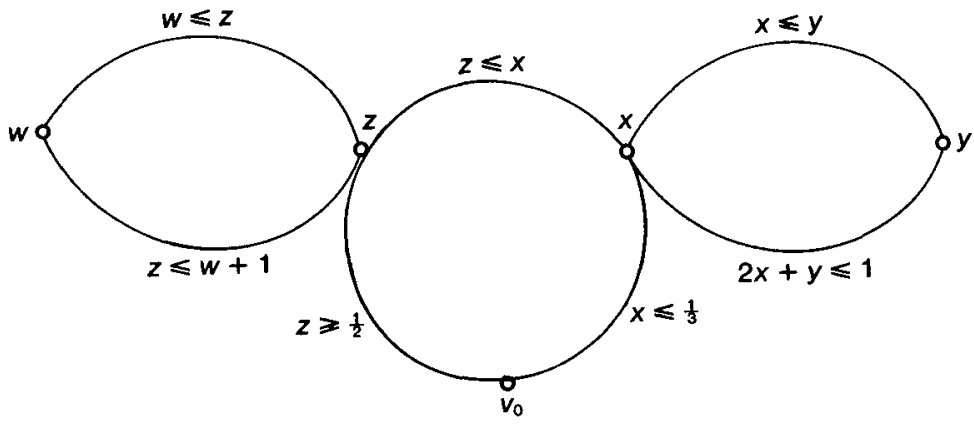

Fig 2. Closure of $G$

Definition. Let $G$ be the graph for $S$. Obtain a closure $G^{\prime}$ of $G$ by adding, for each simple admissible loop $P$ (modulo permutation and reversal) of $G$ a new edge labeled with the residue inequality of $P$.

Note that closures are not necessarily unique, since the initial vertex of each permutable loop can be chosen arbitrarily.

THEOREM. $S$ is unsatisfiable if and only if $G^{\prime}$ has an infeasible simple loop.

Figure 2 shows the unique closure of the graph of Figure 1. Note that the only loop of $G$ contributing an edge to $G^{\prime}$ is the $x y x$ loop. The $v_{0} x z v_{0}$ loop of $G^{\prime}$ is infeasible (having residue $\left(0,0,-\frac{1}{3}\right)$ ); hence the example $S$, according to the theorem, must be unsatisfiable.

We show later that any cyclic permutation of an infeasible permutable loop is itself infeasible, and that the reverse of an infeasible loop is also infeasible. We thus have the following decision procedure for satisfiability of $S$ :

(1) The simple admissible loops of $G$ are enumerated modulo cyclic permutation and reversal, and their residues are computed. If any loops are found to be infeasible, $S$ is unsatisfiable.

(2) Otherwise, the closure of $G$ is formed by adding a new edge for each residue inequality. The residues of all newly formed simple admissible loops are now computed. If any are found to be infeasible, $S$ is unsatisfiable. Otherwise $S$ has solutions.

Note that this procedure, as stated, does not actually construct a solution if $S$ is feasible. The proof of the main theorem, given in Section 7, provides such a 
construction. Note also that the new admissible loops formed in (2) must have initial vertex $v_{0}$.

\section{Efficiency and Other Issues}

Any implementation of the procedure must, of course, incorporate some means of generating the simple loops of a graph. For this purpose, several algorithms exist (Johnson [14], Read and Tarjan [21], Szwarcfiter and Lauer [25]) that operate in time order $l(|V|+|E|)$, and space order $|V|+|E|$, where $l$ is the number of loops generated. These algorithms are easily modified to generate only admissible loops without adversely affecting efficiency. Since each loop has length on the order of $|V|$, these algorithms require little more time than that needed for output. A graph may, of course, have quite a few simple loops-exponentially many (in $|E|$ ), in fact, in the worst case. One can show that the procedure we have described, like the simplex method, exhibits exponential worst case asymptotic behavior.

In practice, however, one does not encounter such behavior. The sets of inequalities that arise from verification conditions usually have the form of transitivity chains. The corresponding graphs are treelike, seldom having more than a few loops. Most of the loops that do occur are 2-loops, which are easily tested at the time the graph is constructed.

Pratt [20] has noted that these sets often fall within what he has termed separation theory. All the inequalities of such sets are of the form $x \leq y+c$. The residue of a loop whose labeling inequalities are of this form is given by one of $\langle 1,-1, m\rangle$, $\langle-1,1, m\rangle$, where $m$ is the sum of the constants $c$ around the loop. The graph for a set $S$ in separation theory is thus its own closure, so the main theorem of the last section reduces, in this case, to Pratt's observation that such a set $S$ is infeasible if and only if the sum of the constants around some simple loop is negative. Pratt notes that this condition can be tested in order $(|V|+|E|)^{3}$ time by taking a max $/+$ transitive closure of the incidence matrix of the graph. In practice, however, it may be more efficient to generate loops using one of the algorithms mentioned earlier.

Note that a set of inequalities in separation theory with integer constants is integer feasible if and only if it is real feasible. While the main theorem therefore decides integer feasibility in this case, it cannot decide integer feasibility in general. It has been observed [23], however, that the transformations Bledsoe [4] describes for reducing formulas in integer arithmetic to sets of inequalities tends to produce sets that are integer feasible if and only if they are real feasible. The main theorem thus provides a useful, but not complete, test for integer feasibility.

\section{Strict Inequalities}

The procedure is trivially generalized to handle strict inequalities (i.e., inequalities of the form $a x+b y<c$ ). Let an admissible loop be strict if one or more of its edges is labeled with a strict inequality. A strict loop $P$ with residue $\left\langle a_{P}, b_{P}, c_{P}\right\rangle$ is infeasible if $a_{P}+b_{P}=0$ and $c_{P} \leq 0$. If the definition of closure is now modified in such a way that new edges arising from strict loops are labeled with strict inequalities, the main theorem still holds.

\section{Extension to Arbitrary Sets of Inequalities}

The method can be further generalized to sets of inequalities with arbitrary coefficients and arbitrary numbers of variables. The basic idea is illustrated by the following example. Consider the set

$$
S=\{x \leq y, y \leq z, z \leq y-x+1, x \geq 2\} .
$$




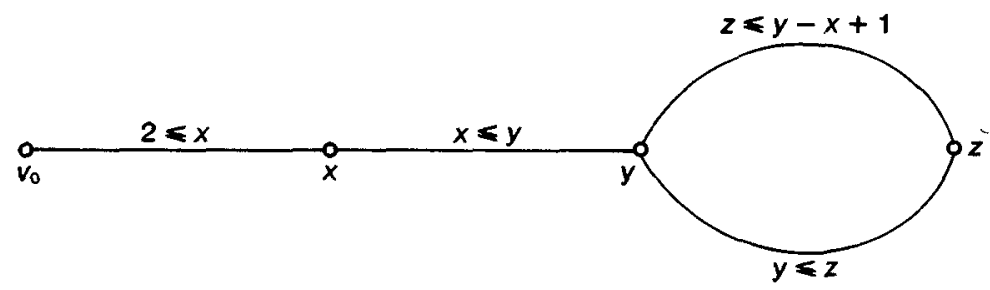

Fig. 3. Graph $G$ for $\{x \leq y, y \leq z, z \leq y-x+1, x \geq 2\}$.

Note that the inequality $z \leq y-x+1$ has three variables. As shown in Figure 3, we choose two of the three (say $z$ and $y$ ) as the endpoints of the edge corresponding to this inequality in the graph $G$ for $S$. The term $(-x+1)$ becomes the "constant" of this inequality. The residue of the only simple loop $(y z y)$ is given by

$$
\langle 1,-1,0\rangle *\langle 1,-1,-x+1\rangle
$$

and is computed "symbolically" to obtain $\langle 1,-1,-x+1\rangle$. Note that this loop is infeasible unless $-x+1 \geq 0$. If the residue inequality $-x+1 \geq 0$ is now added to the graph, an infeasible simple loop $\left(v_{0} x v_{0}\right)$ results, thus making $S$ unsatisfiable.

We now describe the procedure for an arbitrary set $S$. We assume that the variables of $S$ other than $v_{0}$ are ordered in some way. Each variable that is the lowest or second lowest ranked variable in every inequality in which it appears is said to be a primary variable. We adopt the convention that the edge corresponding to a given inequality is always attached to the two nodes corresponding to its primary variables. If it has only one primary variable, one end is attached to $v_{0}$, and if it has no primary variables, both ends are attached to $v_{0}$. The procedure is as follows:

(1) Compute a closure $G^{\prime}$ of the graph $G$ for $S$ as usual, evaluating residues "symbolically" as in the example. If $G^{\prime}$ has an infeasible loop, terminate returning "unsatisfiable." Otherwise, if all the variables of $S$ are primary, terminate returning "satisfiable."

(2) Otherwise, repeat the procedure using the set of residue inequalities of $G^{\prime}$ in place of $S$.

Note that the procedure must terminate since the number of nonprimary variables must decrease each iteration. One can prove as an extension of the main theorem that the general procedure is complete as well as sound.

R. Tarjan $^{3}$ has observed that any set of inequalities can be polynomial transformed to one with no more than three variables per inequality through the addition of new variables. The inequality $w+x+y+z \leq 1$, for example, is replaced by $w+x \leq v$, $w+x \geq v, v+y+z \leq 1$. For sets with inequalities having no more than three variables, only two iterations of the procedure are ever required. There does not seem to be any fast way to transform a set of inequalities to one having inequalities with no more than two variables.

\section{Proof of the Main Theorem}

It follows from Lemma 1 and from the definition of closure that a set $S$ of inequalities (each having no more than two variables) is satisfiable if and only if $S^{\prime}$ is unsatisfiable, where $S^{\prime}$ labels the edges of a closure of the graph for $S$. If we define a closed graph as one that is a closure of itself, the main theorem can thus be restated as follows.

\footnotetext{
${ }^{3}$ Private communication.
} 
THEOREM. If $G$ is a closed graph for $S$, then $S$ is satisfiable if and only if $G$ has no infeasible simple loop.

The proof of the theorem requires a number of technical lemmas. Proofs are omitted for the more trivial of these.

Notation. Where $P$ and $Q$ are paths, let $P Q$ denote the concatenation of $P$ with $Q$.

LEMMA 2. If $P$ and $Q$ are admissible paths, then $P Q$ is admissible if and only if $b_{Q}$ and $a_{P}$ are of opposite sign.

Notation. Let $T=\langle a, b, c\rangle$ be a triple of reals. Then $T^{\sim}$ denotes the triple $\langle b, a, c\rangle$.

Lemma 3. If $T_{1}, T_{2}$ are triples, $T_{1} * T_{2}=\left(\widetilde{T}_{2} * \widetilde{T}_{1}\right)$.

COROLLARY 4. If $Q$ is the reverse of an admissible path $P$, then $r_{P}=r \tilde{Q}$.

COROLlARY 5. The reverse of an infeasible loop is itself infeastble.

Lemma 6. Any permutation of an infeasible permutable loop is infeasible.

Proof. Say $P$ is infeasible and $P^{\prime}$ is a permutation of $P$. Then there are paths $Q$ and $R$ such that $P=Q R$ and $P^{\prime}=R Q$. Thus,

$$
r_{P}=\left\langle k a_{Q} a_{R},-k b_{Q} b_{R}, k\left(c_{Q} a_{R}-c_{R} b_{Q}\right)\right\rangle
$$

and

$$
r_{P^{\prime}}=\left\langle k^{\prime} a_{R} a_{Q},-k^{\prime} b_{R} b_{Q}, k^{\prime}\left(c_{R} a_{Q}-c_{Q} b_{R}\right)\right\rangle,
$$

where $k=a_{R} /\left|a_{R}\right|$ and $k^{\prime}=a_{Q} /\left|a_{Q}\right|$. Note that by admissibility of $P$ and $P^{\prime}$, both $a_{R}$ and $a_{Q}$ are nonzero. By infeasibility of $P, a_{R} a_{Q}-b_{Q} b_{R}=0$ and $k\left(c_{Q} a_{R}-c_{R} b_{Q}\right)<0$. Therefore,

$$
a_{R}\left(\frac{c_{Q} b_{R} b_{Q}}{a_{Q}}-c_{R} b_{Q}\right)<0
$$

therefore,

$$
a_{R} b_{Q}\left(\frac{c_{Q} b_{R}}{a_{Q}}-c_{R}\right)<0
$$

therefore,

$$
c_{R}-\frac{c_{Q} b_{Q}}{a_{Q}}<0 \quad \text { (since } a_{R} \text { and } b_{Q} \text { have opposite signs); }
$$

and therefore,

$$
k^{\prime}\left(c_{R} a_{Q}-c_{Q} b_{R}\right)<0 .
$$

Recalling that $a_{R} a_{Q}-b_{Q} b_{R}=0$, we thus have that $P^{\prime}$ is infeasible. Q.E.D.

Notation. Where $u, v, w$ are reals, let $u<^{w} v$ mean that $u<v$ if $w \geq 0$ and $u>v$ if $w<0$.

Definttion. Where $P$ is an admissible path, the discriminant $d_{P}$ of $P$ is given by $c_{P} /\left(a_{P}+b_{P}\right)$.

Note that an infeasible loop is one with discriminant $-\infty$. 
LEMMA 7. If $P Q$ is an admissible loop from $v_{0}$ to $v_{0}$, then $P Q$ is infeasible iff $d_{P}>^{a_{Q}} d_{Q}$ iff $d_{P}<{ }^{a_{P}} d_{Q}$.

Notation. In the following, let $\left\langle a_{1}, b_{1}, c_{1}\right\rangle,\left\langle a_{2}, b_{2}, c_{2}\right\rangle,\left\langle a_{3}, b_{3}, c_{3}\right\rangle$, and $\left\langle a_{P}, b_{P}, c_{P}\right\rangle$ denote the residues of $P_{1}, P_{2}, P_{3}$, and $P$, respectively.

LEMMA 8. If $G$ is closed and has an infeasible loop from $v_{0}$ to $v_{0}, G$ then has an infeasible simple loop.

Proof. Let $P$ be a shortest infeasible loop from $v_{0}$ to $v_{0}$ in $G$. If $P$ is simple, we are done. Otherwise, since by admissibility the intermediate vertices of $P$ are distinct from $v_{0}, P$ can be expressed as $P_{1} P_{2} P_{3}$, where $P_{2}$ is simple. We claim that $P_{2}$ is also infeasible.

Suppose it is not. Then either $a_{2}+b_{2}=0$ and $c_{2} \geq 0$ or $d_{P_{2}}$ is finite. in the former case, $a_{2}$ and $b_{2}$ have opposite signs. It follows from Lemma 2 that $b_{1}$ and $a_{3}$ must have opposite signs as well, hence $P_{1} P_{3}$ is admissible. Now since

$$
r_{P_{1} P_{2}}=\left\langle 0, b_{1}, c_{1}\right\rangle *\left\langle a_{2}, b_{2}, c_{2}\right\rangle=\frac{a_{2}}{\left|a_{2}\right|}\left\langle 0,-b_{1} b_{2}, c_{1} a_{2}-c_{2} b_{1}\right\rangle,
$$

we have

$$
d_{P_{1} P_{2}}=\frac{c_{1} a_{2}-c_{2} b_{1}}{-b_{1} b_{2}}=\frac{c_{2}}{b_{2}}-\left(\frac{a_{2}}{b_{2}}\right) d_{p_{1}}=\frac{c_{2}}{b_{2}}+d_{P_{1}}
$$

Since $P$ is infeasible, we have from Lemma 7 that

$$
\frac{c_{2}}{b_{2}}+d_{P_{1}}>^{a_{3}} d_{P_{3}}
$$

Thus,

$$
c_{2}+b_{2} d_{P_{1}}>^{a_{3} b_{2}} b_{2} d_{P_{3}}
$$

therefore

$$
c_{2}+b_{2} d_{P_{1}}<b_{2} d_{P_{3}} \quad \text { (since } a_{3} \text { and } b_{2} \text { have opposite signs); }
$$

therefore,

$$
b_{2} d_{P_{1}}<b_{2} d_{P_{3}} \quad\left(\text { since } c_{2} \geq 0\right)
$$

therefore,

$$
d_{P_{1}}<^{b_{2}} d_{P_{3}}
$$

and therefore,

$$
d_{P_{1}}>^{a_{3}} d_{P_{3}} \quad \text { (since } b_{2} \text { and } a_{3} \text { have opposite signs). }
$$

But then $P_{1} P_{3}$ is infeasible by Lemma 2 , contradicting our assumption that $P$ is the shortest such loop.

Now if $d_{P_{2}}$ is finte, the closedness of $G$ provides that some vertex $x$ on $P_{2}$ must be connected to $v_{0}$ via an edge $E$ labeled $a x \leq c$, where $c / a$ is the discriminant of some cyclic permutation $P_{2}^{\prime}$ (possibly $=P_{2}$ ) of $P_{2}$. We then have three cases.

Case 1: $P_{2}$ is not permutable. Then $P_{2}^{\prime}=P_{2}, a=a_{2}+b_{2}, c=c_{2}$, and by Lemma 2, $a_{2}$ and $b_{2}$ are of the same sign. Also, $a$ must be of this sign; hence both $P_{1} E$ and $E P_{2}$ are admissible. An argument similar to the one above gives the result that one or the other of $P_{1} E, E P_{2}$ must be infeasible, contradicting the shortness of $P$. 
Case 2: $P_{2}$ is permutable and $P_{2}^{\prime}=P_{2}$. In this case we have from Lemma 2 that $a_{2}$ and $b_{2}$ have opposite signs; hence $b_{1}$ and $a_{3}$ do as well. An argument similar to that given earlier shows that one of $P_{1} P_{3}, P_{1} E$, and $E P_{2}$ must be infeasible, again contradicting the shortness of $P_{2}$.

Case 3: $P_{2}$ is permutable and $P_{2}^{\prime} \neq P_{2}$. Let $P_{4}$ be the initial subpath of $P_{2}$ which terminates at $x$, and let $P_{5}$ be the final subpath of $P_{2}$ which originates at $x$ (so that $P_{2}=P_{4} P_{5}$ ). In this case it can be shown that $P_{1} P_{3}$ is admissible, that one of $P_{1} P_{4} E, E P_{5} P_{3}$ is admissible, and that one of these three paths must be infeasible. The shortness of $P$ is thus once again contradicted. Q.E.D.

THEOREM. Let $G$ be a closed graph for $S$. Then $S$ is satisfiable if and only if $G$ has no simple infeasible loop.

Proof. It follows from Lemma 1 that if $G$ has a simple, infeasible loop, $S$ must be unsatisfiable. Conversely, suppose $G$ has no such loop. We will show that $S$ is satisfiable by constructing a solution.

Let $v_{1}, \ldots, v_{r}$ be the variables of $S$ other than $v_{0}$. We construct a sequence $\hat{v}_{0}$, $\hat{v}_{1}, \ldots, \hat{v}_{r}$ of reals and a sequence $G_{0}, G_{1}, \ldots, G_{r}$ of graphs inductively as follows:

(1) Let $\hat{v}_{0}=0$ and $G_{0}=G$.

(2) Suppose $\hat{v}_{\imath}$ and $G_{l}$ have been determined for $0 \leq i<j \leq r$. Let

$\sup _{J}=\min \left\{d_{P} \mid P\right.$ is an admissible path from $v_{J}$ to $v_{0}$ in $G_{J-1}$ and $\left.a_{P}>0\right\}$, inf $=\max \left\{d_{P} \mid P\right.$ is an admissible path from $v_{0}$ to $v_{J}$ in $G_{j-1}$ and $\left.b_{P}<0\right\}$.

(where it is understood that $\min \varnothing=\infty$ and $\max \varnothing=-\infty$ ). Then let $\hat{v}_{J}$ be any value in the interval $\left[\inf _{j}, \sup _{J}\right]$. (We show momentarily that inf $\mathrm{f}_{J} \leq \sup _{J}$.) Let $G_{J}$ be obtained from $G_{J-1}$ by adding two new edges from $v_{j}$ to $v_{0}$, labeled $v_{J} \leq \hat{v}_{j}$ and $v_{j} \geq \hat{v}_{J}$, respectively.

To ensure that the $\hat{v}_{j}$ and $G_{j}$ are well defined, we must show that for $1 \leq j \leq r$, inf $_{J} \leq \sup _{J}$. It will then remain to show that the $\hat{v}_{J}$ do indeed give a solution for $S$.

We need the following claim.

\section{Claim}

(i) For $1 \leq j \leq r, \operatorname{lnf} f_{j} \leq \sup _{j}$.

(ii) For $0 \leq j \leq r, G$, has no infeasible simple loops.

Proof. By induction on $j$.

Basis. $j=0$. In this case, (i) holds vacuously, and (ii) holds since $G_{0}=G$.

Induction Step. $0<j \leq r$.

For (i), suppose, to the contrary, that inf $>\sup _{J}$. Then in $G_{J-1}$, admissible paths $P_{1}, P_{2}$ exist from $v_{0}$ to $v_{j}$ and $v_{j}$ to $v_{0}$, respectively, with $b_{P_{1}}<0, a_{P_{2}}>0$, and $d_{P_{1}}>d_{P_{2}}$. By Lemma $2, P_{1} P_{2}$ is an admissible loop, and by Lemma $7, P_{1} P_{2}$ is infeasible. By Lemma 8 , then, $G_{j-1}$ has a simple infeasible loop, contradicting (ii) of the induction hypothesis.

For (ii), suppose $G_{J}$ has an infeasible simple loop $P$. Since $G_{J-1}$ has no such loop, and since the loop formed by the two new edges added to $G_{J-1}$ to obtain $G_{J}$ is not infeasible, $P$ (or its reverse) must be of the form $P^{\prime} E$, where $E$ is one of the two new edges (say the one labeled $v_{J} \leq \hat{v}_{j}$; the other case is handled similarly), and $P^{\prime}$ is a path from $v_{0}$ to $v_{j}$ in $G_{J-1}$. But then, by Lemma $7, d_{P^{\prime}}>d_{E}=\hat{v}_{y}$, contradicting $\hat{v}_{j} \geq$ inf $f_{j} \geq d_{P^{\prime}}$ (Note that $b_{P^{\prime}}<0$ from the admissibility of $P^{\prime} E$ ). Q.E.D. 
It now remains to show that the $\hat{v}_{J}$ satisfy $S$. So let $a x+b y \leq c$ be an inequality of $S$. We claim that $a \hat{x}+b \hat{y} \leq c$. We treat the case in which $a>0$ and $b>0$; the other cases are argued similarly. Let $E$ be the edge labeled $a x+b y \leq c$ in $G_{r}$. Then, where $E_{1}$ is the edge labeled $\hat{x} \leq x$ in $G_{r}$ and $E_{2}$ is the one labeled $y \leq \hat{y}, E_{1} E E_{2}$ forms an admissible loop. The residue of this loop is

$$
\langle 0,-1,-\hat{x}\rangle \cdot\langle a, b, c\rangle *\langle 1,0, \hat{y}\rangle=\langle 0,0,-a \hat{x}-b \hat{y}+c\rangle .
$$

Since, by the claim proved above and Lemma $8, G_{r}$ has no infeasible loops from $v_{0}$ to $v_{0}$, we have $-a \hat{x}-b \hat{y}+c \geq 0$. Thus $a \hat{x}+b \hat{y} \leq c$, as required. Q.E.D.

ACKNOWLEDGMENTS. The author gratefully acknowledges the insights provided by R. Tarjan and M. W. Green.

\section{REFERENCES}

(Note References $[2,5-7,10-13,15,17,19,24,26]$ are not cited in the text )

1. Apsvall, B., AND Shiloach, Y A polynomial-tıme algorithm for solving systems of linear equalities with two variables per inequality Proc 20th Ann Symp on Foundations of Computer Science, San Juan, Puerto Rico, 1979, pp 205-217 Also to appear in SIAM J Comput

2 Bledsoe, W W Program correctness Memo ATP-14, Mathematıcs Dep, Unıv of Texas, Austın, Texas, Jan 1974

3 Bledsoe, W W. The sup-inf method in Presburger arithmetıc Memo ATP-18, Mathematıcs Dep, Untv. of Texas, Austın, Texas, Dec. 1974.

4 Bledsoe, W W A new method for proving certain Presburger formulas Advance Papers, 4th Int Joint Conf on Artıficial Intelligence, Tibilıst, Russia, Sept. 1975, pp 15-21

5 Bledsoe, W W, Boyer, R S, and Henneman, W H Computer proofs of limit theorems Artif Intell 3 (1972), 27-60

6 BLEDSOE, W W., AND BRuell, P A man-machine theorem-proving system Artif Intell 5 (1974), $51-72$

7. COOPER, D C. Programs for mechanıcal program verification In Machine Intelligence 6, B Meltzer, Ed, American Elsevier, New York, 1971, pp 43-59

8 Dantzig, G B Linear Programming and Extensions Princeton University Press, Princeton, N J, 1962

9. Deutch, L P An interactive program verifier Ph D Dissertation, Unıv of California, Berkeley, Calıf., 1973

10. Elspas, B., Boyer, RE, Shostak, R, and Spitzen, J A venfication system for JOVIAL/J3 programs Tech Rep 3756-1, Stanford Research Institute. Menlo Park, Calıf, Jan 1976.

11. Gomory, RE An algorithm for integer solutions to linear programs Princeton IBM Math Res Report, Nov 1958 Also in Recent Advances in Mathematcal Programming, R L Graves and P Wolfe, Eds, McGraw-Hill, N Y., 1963, pp 269-302

12 Good, D.I, London, R L, AND Bledsoe. W W An interactive verification system Proc 1975 Int Conf on Reliable Software, Los Angeles, Calif, April 1975, pp 482-492

13 Igarashi, S, LoNDON, R L, AND LUCKHAM, D C Automatic program verification I A logical basis and its implementation Stanford AI Memo 200. Stanford, Calif, May 1973, and USC Information Sciences Instıtute Rep ISI/RR-73-11, Unıv of Southern Calıfornıa, Los Angeles, Calıf, May 1973

14 Johnson, D B Finding all the elementary circuits of a directed graph SIAM J Comput 4 (1975), $77-84$

15 King, J A program verifier Ph D Dissertation, Carnegie-Mellon Unıv, Pitsburgh, Pa, 1969

16 KHachiYAN, L.G A polynomial algorithm in linear programming. Dokl Akad Nauk SSSR Nov Ser 244, 5 (1979), 1093-1096 [Eng trans in Sov Math Dokl 20, 1 (1979), 191-194]

17 LEE, R D. An application of mathematical logic to the integer linear programming problem Notre Dame J Formal Logic XIII, 2 (April 1972)

18 Litvintchouk, S D, and Pratt, V R. A proof checker for dynamic logic 5 th Int Joint Conf on Artıficial Intelligence, Boston, Mass, August 1977, pp 552-558

19 Mateti, P., and Prabhaker. M On algorithms for enumerating all circuits of a graph SIAM $J$ Comput 5 (March 1976), 90-99

20 Pratt, V R Two easy theories whose combination is hard. Tech. Rep, Massachusetts Institute of Technology, Cambridge, Mass., Sept 1977 
21. READ, R.C, AND TARJAN, RE. Bounds on backtrack algonthms for listing cycles, paths, and spanning trees. ERL Memo M 433, Electronıcs Research Lab, Univ. of California, Berkeley, Calif, 1973.

22 Shostak, R. An efficient decision procedure for arithmetic with function symbols. Presented at 5 th Int. Joint Conf. on Artıficial Intelligence, Cambridge, Mass., Aug 1977.

23 Shostak, R. On the SUP-INF method for proving Presburger formulas. J. ACM 24, 4 (Oct 1977), $529-543$

24. SuzuKı, N. Verifyıng programs by algebraic and logical reduction Proc. 1975 Int. Conf. on Reliable Software, Los Angeles, Calıf., April 1975, pp 473-481.

25. Szwarcfiter, J.L, and Lauer, P E Finding the elementary cycles of a directed graph in $O(n+m)$ per cycle. Tech. Rep No. 60, Univ. of Newcastle upon Tyne, Newcastle upon Tyne, England, May 1974

26 TARJAN, R. Enumeration of the elementary circuits of a directed graph SIAM J. Comput. 2,3 (Sept. 1973), 211-216.

27. Waldinger, R J , AND Levitt, K.N. Reasoning about programs. J. Arty Intell. 5 (1974), 235-316.

RECEIVED MAY 1979; REVISED MAY 1980, ACCEPTED JUNE 1980 